\title{
A Systematic Review: Predict the COVID-19 Severity by HDL and Triglycerides Level
}

Hoo Yumilia, Daniel Setiawan Nathan*

Division of Endocrinology, Diabetes, and Metabolism, Departement of Internal Medicine, Faculty of Medicine, Maranatha Christian University/Immanuel Hospital,Bandung

*Corresponding author: Daniel Setiawan Nathan, Division of Endocrinology, Diabetes, and Metabolism, Departement of Internal Medicine, Faculty of Medicine, Maranatha Christian University/Immanuel Hospital,Bandung.

Received Date: June 07, 2021; Accepted Date: June 25, 2021; Published Date: July 12, 2021.

Citation: H Yumilia, Daniel S Nathan. (2021) A Systematic Review: Predict the COVID-19 Severity by HDL and Triglycerides Level. Journal of Endocrinology and Disorders. 5(4): Doi:10.31579/2692-9759/076

Copyright: (c) 2021 Daniel Setiawan Nathan. This is an open-access article distributed under the terms of the Creative Commons Attribution License, which permits unrestricted use, distribution, and reproduction in any medium, provided the original author and source are credited.

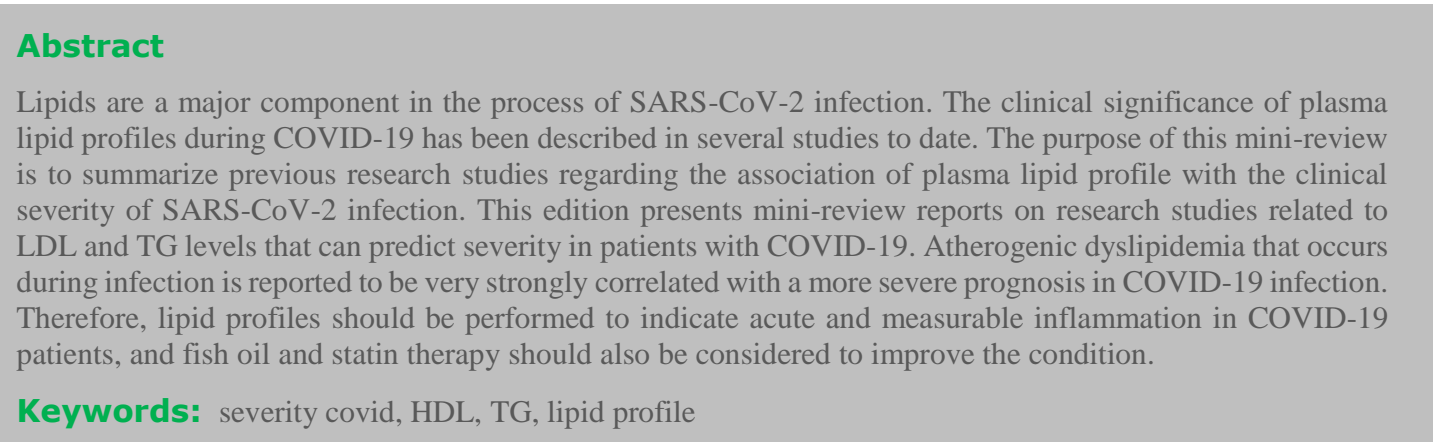

\section{Introduction}

The actual pathophysiological mechanism of COVID-19 has not been fully identified, so the COVID-19 pandemic has spread rapidly throughout the world and is still far from under control. While treatment options are very limited, identifying associated risk factors is very important to improve the prognosis of Covid-19 patients. Identity, age, gender, therapeutic background, and comorbidities are the main clinical determinants related to the severity of Covid-19 patients [1-3]. In addition, factors such as elevated blood pressure and dysglycemia are strongly correlated with a poorer prognosis. Recently, early lipid dyslipidemia has become one of the main cardiovascular risk factors that correlate with the prognosis of Covid-19 patients. This paper will systematically summarize the dyslipidemias that play a role in the severity of SARS-CoV-24 infection.

Lipids are a major component in the infectious process because they are structurally important in membranes of cellular and subcellular organelles-lipid membrane components act in regulating the trafficking of transmembrane molecules, including infectious agents for viruses. Internalization of the virus requires the attachment of the virus to the membrane of the host cell; then, it will activate the mechanism of endocytosis [5]. The lipid composition of the membrane and especially the lipid raft plays an important role in this process [6]. When it is inside the cell, the virus will replicate and use the cell's metabolic machinery, which has been invaded [7]. New viral particles will be synthesized out of the cell by passing through the lipid-rich cell membrane. Previous research has been carried out using animal model studies; intracellular cholesterol can significantly increase the infectivity of cellular SARS- CoV-2 [8]. And recent research also suggests that the C-terminal SREBP-2 fragment found in blood samples from COVID-19 patients was detected to play a role as a marker of the severity of the diagnosis of Covid-19 patients - therapeutic targets to prevent cytokine storms and organ damage [9]. In the host cell, the virus will be modified for cell metabolism by changing energy production pathways for its own sake. Both cellular glucose well as lipid metabolism will be extensively modified6, and metabolomics studies in SARS-CoV-2-infected patients have outlined specific lipidomic fingerprints recently [10].

Structural functions and energy supply, intracellular lipids also act as molecules that give intracellular signals, or the term is a transcription factor. Viral particles also impair this pathway by altering cell physiology and carrying out the process of apoptosis or cell death.

Clinical trials have been carried out to calculate the rate of changes in lipid metabolism based on the results of cholesterol measurements on lipoprotein particles and triglyceride levels that have been tested. Unfortunately, researchers have not determined how this parameter mechanism can play an essential role in cellular lipids in viral infection. This infectious disease is highly correlated with low concentrations of HDL cholesterol (HDL-C) and low concentrations of LDL cholesterol (LDL-C). Triglyceride levels were also stable and even slightly increased $[6,11]$. Sufficiently low HDL-C levels and high TG levels 
have been correlated as effective biomarkers in increasing the risk for these infections [12]. Associated with the presence of SARS- CoV-2 infection and LDL levels-C, HDL-C, and triglycerides (TG) have been described as important in measuring the severity of infection 6 and the role of these lipids in immune mechanisms or the immune system [13].

Dyslipidemia that occurs during infection is closely related to the severity of patients with COVID-19 [14]. Therefore, it is hypothesized that low plasma lipid levels are currently considered a consequence of hypermetabolic and nutritional deficiencies in patients infected with the Covid-19 virus. Theories regarding metabolic pathways related to the immune response and infection play a role in this transformation process [11]. Inflammatory mediators, lipids, cytokine-modifying lipids, and intermediate classes of lipids are also produced during infection that interferes with several lipid metabolism pathways by reducing clearance of triglyceride-rich lipoproteins or reducing lipid metabolism. In addition, the synthesis of apolipoprotein (apo) A1 reduces the synthesis and absorption of cholesterol.

Overall, we hypothesize that this systematic review of lipid metabolism analyzes important considering that screening lipid profile evaluation should be considered a parameter for evaluating the prognosis of patients infected with SARS-CoV-2 toobtain adequate treatment, and the outcome will be significant.

\section{Objective}

This mini-review aims to summarize cases that occurred among Covid-19 patients who reported cases of standard serum lipid concentrations, namely HDL and TG, withcorrelations of the severity of COVID-19 infection. Some of the severity levels of cases in this mini-review were mild if the patient was without oxygen therapy or only given oxygen with a mask or nasal prong. Meanwhile, cases were categorized as severe when the patient needed additional ventilation or special therapy to support organ repair function in the ICU or also a case of death.

\section{Methods}

Collect data or sources related to the topic raised in a journal and other studies. For example, there are seven journals about alterations of lipid profile in predict the severity of COVID-19.

\section{Result}

The role of lipids in cell biology is that of the backbone of cell membranes, in cellular interconnection, membrane trafficking, energy sources, and heat insulation [21]. They are necessary for viruses to transport host cell membranes. And it is known that viral infection alters lipid metabolism in favor of SARS-CoV-2 virus replication. Lipid studies have revealed a recent theory that coronaviruses significantly alter the lipid composition of infected cells [22]. Viruses use and alter lipid signaling and metabolism for their replication advantage because lipids form the main structure of membranes and play a very important role in energy sources and agents-intercellular signaling [23].

Replication of enveloped viruses such as SARS-CoV-2- which enters cells through a process called endocytosis and uses intracellular organelles to produce different parts requires the main source of lipids [24]. Therefore, studying how SARS-COV-2 infection affects metabolism and lipid profile may explain the correlation between lipid profile and the inflammatory process during CoViD-19.

\begin{tabular}{|c|c|c|c|}
\hline No & Reserchers & $\begin{array}{c}\text { Journal Name } \\
\text { and Year } \\
\text { Publication }\end{array}$ & Resut \\
\hline 1 & $\begin{array}{l}\text { Hu X, Chen D, } \\
\text { Wu L, He G, Ye } \\
\text { W. }\end{array}$ & $\begin{array}{l}\text { Clin } \\
\text { Acta. } 2020\end{array}$ & $\begin{array}{c}\text { A cross-sectional analysis study of } 1411 \text { hospitalized patients } \\
\text { with a diagnosis of COVID-19, which provides evidence that } \\
\text { low HDL-C and high TG before infection and admission to the } \\
\text { ED are significant indicators of disease severity and are } \\
\text { moderately correlated with ferritin } \\
\text { and D levels. -High dimer. }\end{array}$ \\
\hline 2 & $\begin{array}{lr}\text { Masana } & \text { L, } \\
\text { Correig } & \mathrm{E}, \\
\text { Ibarretxe } & \mathrm{D}, \\
\text { Anoro } & \mathrm{E}, \\
\text { Arroyo } & \mathrm{JA}, \\
\text { Jericó C. } & \end{array}$ & Sci Rep. 2021 & $\begin{array}{c}\text { This research provides insight that TC and LDL-C levels are low } \\
\text { among all patients with COVID-19. And the LDL- C condition } \\
\text { showed a decreasing level as the disease progressed. And most } \\
\text { importantly, HDL-C levels showlower values and the } \\
\text { abnormality occurs in critical } \\
\text { cases. }\end{array}$ \\
\hline 3 & $\begin{array}{l}\text { Wang G, } \\
\text { Zhang Q,Zhao } \\
\text { X, DongH, Wu } \\
\text { C, Wu F. }\end{array}$ & $\begin{array}{l}\text { Lipids Health } \\
\text { Dis. 2020;19(1) } \\
\text { :204. }\end{array}$ & $\begin{array}{l}\text { Research conducted on } 228 \text { cases of COVID-19 that occurred in } \\
\text { China. This study confirmed their hypothesis that LDL-C, HDL- } \\
\text { C, and TC were lower in patients compared to their controls, } \\
\text { namely healthy humans.And } \\
\text { lower HDL-C levels at admission were poor prognostic } \\
\text { factors associated with negative disease outcomes. }\end{array}$ \\
\hline 4 & $\begin{array}{l}\text { Tanaka S, De } \\
\text { Tymowski } \mathrm{C}, \\
\text { Assadi M, } \\
\text { Zappella } \mathrm{N}, \\
\text { Jean-Baptiste S, } \\
\text { Robert T. }\end{array}$ & $\begin{array}{l}\text { PLoS } \\
\text { One. } 2020 ; 15(9)\end{array}$ & $\begin{array}{l}\text { This research is a study of lipid profile trends in COVID- } 19 \\
\text { patients admitted to the ICU. This study also presented data that } \\
\text { all patients had low levels of LDL-C and HDL-C. Unfortunately, } \\
\text { this problem is not a predictor of mortality, lower cholesterol } \\
\text { levels at the onset of ventilator- associated pneumonia are } \\
\text { associated with increased mortality in patients with Covid-19. }\end{array}$ \\
\hline
\end{tabular}




\begin{tabular}{|l|l|l|l|}
\hline 5 & $\begin{array}{l}\text { Song JW, Lam } \\
\text { SM, Fan X, } \\
\text { Cao WJ, } \\
\text { Wang SY, } \\
\text { Tian } \\
\text { Omics-Driven } \\
\text { Metab. 2020;32 } \\
(2): 188-202 . \text { e5 }\end{array}$ & $\begin{array}{l}\text { Prospective studies show lipid changes occurring inCOVID-19 } \\
\text { patients. This study found the theory that Apo- A1 and HDL-C } \\
\text { were inversely related to measures of disease severity such as } \\
\text { inflammatory markers such as IL-6 and CRP and mortality. This } \\
\text { study demonstrated that significantly higher TG levels and } \\
\text { significantly lower TC and LDL-C levels in severe patients than } \\
\text { in milder cases. }\end{array}$ \\
\hline $\mathbf{6}$ & $\begin{array}{l}\text { Wang G, G, } \\
\text { Zhang Q,Zhao } \\
\text { X, DongH, Wu } \\
\text { C, Wu F. }\end{array}$ & $\begin{array}{l}\text { Lipids Health } \\
\text { Dis. 2020;19(1) }\end{array}$ & $\begin{array}{l}\text { This study presents slightly similar cases and shows an inverse } \\
\text { relationship between HDL and LDL with C- reactive protein } \\
\text { (CRP), which is a useful marker of inflammation severity. }\end{array}$ \\
\hline 7 & $\begin{array}{l}\text { Fan J, Wang H, } \\
\text { Ye G, Cao X, } \\
\text { Xu X, Tan W. }\end{array}$ & $\begin{array}{l}\text { Metabolism: } \\
\text { clinical } \\
\text { experimental. 2 } \\
\text { and }\end{array}$ & $\begin{array}{l}\text { This study compared 17 patients who survived with four patients } \\
\text { who died from COVID-19. An interesting factabout the disease, } \\
\text { the researchers found that although LDL levels decreased in both } \\
\text { groups, the more severe and progressive lipid dysregulation } \\
\text { worsened among the } \\
\text { survivors. }\end{array}$ \\
\hline
\end{tabular}

Table 1: Summary Research about alterations of lipid profile in predict severity COVID-19

\section{Discussion}

Plasma lipid concentrations play a significant role in the clinical management of COVID-19 patients. Changes in lipid profile can be used as a guideline for poor prognostic criteria and play a role in the decision-making process to encourage the identification process to obtain early treatment. From our systematic data, we can explain that low HDL-C levels and high triglyceride levels should be considered high-risk markers for patients with COVID-19. Patients with available pre-infectious lipid profiles, high triglyceride levels, and low HDL cholesterol levels were also correlated with a poorer COVID-19 prognosis. Our review supports the functional relevance of lipids, even standard lipid profiles, as markers of global inflammatory status and risk markers for COVID-19 patients. Previous studies have described the association of rapid changes in lipid profiles in response to COVID19 and the progression of the disease [4].

A large gap may also exist between the incidence of dyslipidemia before viral infection and when lipid disturbances were detected during hospitalization. In thestudy conducted by Lluís Masana et al., lower levels of TC, LDL-C, HDL-C, and non-HDL-C occurred in infected patients compared to levels measured before disease exposure. However, TG levels were significantly higher. Hypotheses about this variation have been proposed. Poor nutritional status and the presence of hypercatabolism that occurs during acute infection must play a role. The contradiction with the paradoxical behavior of triglyceride levels does require additional explanation. In patients with severe evolution of lipid levels exhibiting significantly higher triglycerides and lower HDL-C levels, suggesting factors associated with the evolution of COVID 19 influence these lipid parameters. In patients with preinfection lipid profiles, patients with poorer clinical outcomes had an older age. In addition, they had a history of other comorbidities, which might affect the lipid levels of these patients [16, 17].

Although there were no important differences in baseline therapy, including statin [18] therapy, patients with worse COVID-19 exposure received more specialized therapy. The combination of low HDL levels and high triglyceride levels, referred to as atherogenic dyslipidemia, is closely associated with significant qualitative changes in LDL particles, leading to increased levels of small and dense LDL particles. This motive is driven by insulin resistance, which is usually also associated with obesityand diabetes. However, from this review, body mass index and prevalence rates of obesity and diabetes were similar between groups with mild or severe COVID-19 patients.
Several previous studies have confirmed the hypothesis that inflammation and infectious diseases are correlated with significant changes in lipid metabolism [21]. The general pattern is similar to that of other studies, namely low HDL-C levels and normal or even high triglyceride levels for clinical assessment. However, LDL-Clevels can be maintained or even reduced if this is associated with an increased presence of smaller LDL particles.

Although insulin resistance plays an important role in this lipid disorder, the pathophysiology is more complicated. The key enzyme contributing to this major change is lipoprotein lipase 15 (LPL). In addition, several inflammatory mediators interfere with glucose and lipid metabolism [22].

Cytokines and inflammatory mediators are also overexpressed during COVID-19 and can interact directly with LPL or its regulatory proteins, such as apo CII [23]. Several proteins delivered by activated macrophages, such as interleukin (IL)-1, IL-11, Interferon- $\gamma$, and tumor necrosis factor (TNF), as well as products of bacterial origin, such as lipoprotein-associated lipopolysaccharide (LPS) and Modified septicemias such as oxidized LDL or lysophosphatidylcholine have been shown to inhibit LPL activity [15, 21, 24].

Lower LPL activity will decrease triglyceride-rich lipoprotein (TRL) to LDL, which will result in high TG and low HDL levels. In addition, changes in cholesteryl ester transfer protein (CETP) activity leading to smaller LDL particles and lower HDL concentrations have also been described and associated with a poorer infection prognosis. Although several of these pathways are hypothesized to explain low HDL levels, including inhibition of apo A1 synthesis or even increased clearance of HDL due to absorption of inflammatory mediators such as serum amyloid A (SAA)by HDL, they should be considered [11].

Recent plasma lipidomic data from patients infected with SARS-CoV2 have revealed substantial modifications of the lipid family during infection. For example, the plasma concentration of monosialodihexosyl ganglioside was negatively correlated with CD4 $+\mathrm{T}$ cells and positively correlated with disease severity [10]. Interestingly, clinical lipid parameters correlate differently with lipid families. In general, cholesterol levels inapo B and apo A1 lipoproteins tended to show a direct correlation with plasmaphosphatidylcholine levels and extracellular vesicle sphingomyelin levels. In contrast, triglyceride concentrations were more strongly associated with 
phosphatidylethanolamine levels. Although the clinical implications of these observations remain to be fully elucidated, they provide evidence for an association between standard lipids measured at a clinical level and lipid disturbances during COVID-19.

Lipoprotein modifications observed during COVID-19, as in other infectious situations, should not be considered merely as markers of severity or markers of use anti-inflammatory because it may have a pathogenic role. Triglyceride-rich lipoproteins have been associated with innate immunity because of their capacity to bind toxins. In addition, low HDL cholesterol levels can lead to innate immune dysfunction, a first-line defense mechanism against COVID-1929. HDL is an inflammatory mediator that buffers toxic molecules by absorbing them andtransporting them to the liver for clearance [11]. Therefore, the structural and functional changes observed during COVID-19 may influence the evolution of the disease.

This study has several limitations. First, it is a mini-review and thus precludes any analysis of causal relationships in this study. Second, this study cannot confirm the proximity of these concentrations to clinical events in every country with different religions, races, and backgrounds. Similarly, we cannot be sure that past investigators determined exactly when lipid profiles were measured during hospitalization for COVID-19, although it is usually included in the first standard blood test performed after hospital admission; therefore, we can only conclude that the lipid profile was measured in the first 48 h.

The standard lipid concentration is not only a marker of a person's nutritional statusbut has now acted as a biomarker of infection and the degree of inflammation thatmay play a pathophysiological role. In addition, high levels of triglycerides are associated with low HDL$\mathrm{C}$ concentrations and should be considered a marker of poorprognosis. Our review strongly supports the existence of a lipid profile measurement protocol during COVID-19, as the lipid profile appears to be a highly correlated risk biomarker.

\section{Conclusion}

Alteration of lipid metabolism among COVID-19 patients through direct cellular infection mechanisms as well as through systemic inflammatory responses. This resulted in a decrease in HDL-C levels and an increase in TG levels among patients with Covid-19. Lower HDL-C levels appear to have potential as a significant prognostic agent in predicting poor clinical outcome in COVID-19 patients. Therefore, the authors suggest that therapy with fish oil as well as statins targeting these lipid and COVID19 pathomechanisms may have a beneficial role for patients with COVID-19.

\section{References}

1. Grasselli G, et al. (2020) Baseline characteristics and outcomes of 1591 patients infected with SARS-CoV-2 admitted to ICUs of the Lombardy region Italy. JAMA. 323:15741581.

2. Ji W, et al. (2020) Effect of underlying comorbidities on the infection and severity of covid-19 in Korea: a nationwide casecontrol study. J. Korean Med. Sci. 35:1-15.

3. Richardson S, et al. (2020) Presenting characteristics, comorbidities, and outcomes among 5700 patients hospitalized with covid-19 in the New York City area. JAMA. 323:2052-2059.

4. Choi GJ, Kim HM. (2020) The potential role of dyslipidemia in COVID-19 severity: An umbrella review of systematic reviews. J. Lipid Atheroscler. 9:435-442.

5. Wiersinga WJ, Rhodes A, Cheng AC, Peacock SJ, Prescott HC. (2020) Pathophysiology, transmission, diagnosis, and treatment of coronavirus disease 2019 (COVID-19): A review. JAMA. 324:782-793.

6. Abu-Farha M, et al. (2020) The role of lipid metabolism in COVID-19 virus infection and drug target. Int. J. Mol. Sci. 21:3544-3555.

7. Gualdoni GA, et al. (2018) Rhinovirus induces an anabolic reprogramming in host cell metabolism essential for viral replication. Proc. Natl. Acad. Sci. USA.115:7158-7165.

8. Wang H, Yuan Z, Pavel M.A, \& Hansen S.B. (2020) The role of high cholesterol in age-related COVID19 lethality.

9. Lee W, et al. (2020) COVID-19-activated SREBP2 disturbs cholesterol biosynthesis and leads to cytokine storm. Signal Transduct. Target Ther. 5:186-197.

10. Song JW, et al. (2020) Omics-driven systems interrogation of metabolic dysregulation in COVID-19 pathogenesis. Cell Metab. 32:188-202.

11. Trinder M, Boyd JH, Brunham LR. (2019) Molecular regulation of plasma lipid levels during systemic inflammation and sepsis. Curr. Opin. Lipidol. 30:108-116.

12. Saballs M, et al. (2016) HDL-c levels predict the presence of pleural effusion and the clinical outcome of communityacquired pneumonia. Springerplus. 5: 1491-1500.

13. Harris HW, Gosnell JE, Kumwenda ZL. (2000) The lipemia of sepsis: Triglyceride-rich lipoproteins as agents of innate immunity. J. Endotoxin Res. 6:421-430.

14. Wei X, et al. (2020) Hypolipidemia is associated with the severity of COVID-19. J. Clin. Lipidol. 14:297-304.

15. Mead JR, Irvine SA, Ramji DP. (2002) Lipoprotein lipase: Structure, function, regulation, and role in disease. J. Mol. Med. 80:753-769.

16. Sittiwet C, Simonen P, Gylling H, Strandberg TE. (2020) Mortality and cholesterolmetabolism in subjects aged 75 years and older: The Helsinki businessmen study. J. Am. Geriatr. Soc.68:281-287.

17. Orkaby AR. (2020) The highs and lows of cholesterol: A paradox of healthy aging? J. Am.Geriatr. Soc. 68:236-237.

18. Masana L, et al. (2020) Effect of statin therapy on sars-cov-2 infection-related mortalityin hospitalized patients. Eur. Heart J. Cardiovasc. Pharmacother. 1:1.

19. Wu Q, et al. (2017) Altered lipid metabolism in recovered SARS patients twelve years after infection. Sci. Rep. 7:1-12.

20. Saladin R, Vu-Dac N, Fruchart JC, Auwerx J, Staels B. (1996) Transcriptional induction of rat liver apolipoprotein A-I gene expression by glucocorticoids requires the glucocorticoid receptor and a labile cell-specific protein. Eur. J. Biochem. 239:451-459.

21. Yan B, Chu H, Yang D, Sze KH, Lai PM, Yuan S. (2019) Characterization of the Lipidomic Profile of Human Coronavirus-Infected Cells: Implications for Lipid Metabolism Remodeling upon Coronavirus Replication. Viruses.11 (1).

22. Casari I, Manfredi M, Metharom P, Falasca M. (2021) Dissecting lipid metabolismalterations in SARS-CoV-2. Prog Lipid Res.

23. Muller C, Hardt M, Schwudke D, Neuman BW, Pleschka S, Ziebuhr J. Inhibition of Cytosolic Phospholipase A2alpha Impairs an Early Step of Coronavirus Replication in Cell Culture. J Virol.92 (4).

24. V'Kovski P, Al-Mulla H, Thiel V, Neuman BW. (2015) New insights on the role of paired membrane structures in coronavirus replication. Virus Res. 202:33-40. 